\title{
The scaffolding protein NHERF1 sensitizes EGFR-dependent tumor growth, motility and invadopodia function to gefitinib treatment in breast cancer cells
}

\author{
ANTONIA BELLIZZI $^{1}$, MARIA RAFFAELLA GRECO ${ }^{1}$, ROSA RUBINO $^{1}$, ANGELO PARADISO $^{2}$, \\ STEFANIA FORCINITI $^{1}$, KATRINE ZEEBERG ${ }^{1}$, ROSA ANGELA CARDONE ${ }^{1 *}$ and STEPHAN JOEL RESHKIN ${ }^{1 *}$ \\ ${ }^{1}$ Department of Biosciences, Biotechnology and Biopharmaceutics, University of Bari, I-70125 Bari; \\ ${ }^{2}$ Clinical Experimental Oncology Laboratory, National Cancer Research Centre 'Giovanni Paolo II', I-70124 Bari, Italy
}

Received October 9, 2014; Accepted November 26, 2014

DOI: $10.3892 /$ ijo.2014.2805

\begin{abstract}
Triple negative breast cancer (TNBC) patients cannot be treated with endocrine therapy or targeted therapies due to lack of related receptors. These patients overexpress the epidermal growth factor receptor (EGFR), but are resistant to tyrosine kinase inhibitors (TKIs) and anti-EGFR therapies. Mechanisms suggested for resistance to TKIs include EGFR independence, mutations and alterations in EGFR and in its downstream signalling pathways. Ligand-induced endocytosis and degradation of EGFR play important roles in the downregulation of the EGFR signal suggesting that its activity could be regulated by targeting its trafficking. Evidence in normal cells showing that the scaffolding protein $\mathrm{Na}^{+} / \mathrm{H}^{+}$ exchanger regulatory factor 1 (NHERF1) can associate with EGFR to regulate its trafficking, led us to hypothesize that NHERF1 expression levels could regulate EGFR trafficking and functional expression in TNBC cells and, in this way, modulate its role in progression and response to treatment. We investigated the subcellular localization of NHERF1 and its interaction with EGFR in a metastatic basal like TNBC
\end{abstract}

Correspondence to: Professor Stephan J. Reshkin or Dr Rosa A. Cardone, Department of Biosciences, Biotechnology and Biopharmaceutics, University of Bari, Via E. Orabona 4, I-70125 Bari, Italy

E-mail: stephanjoel.reshkin@uniba.it

E-mail: rosaangela.cardone@uniba.it

${ }^{*}$ Contributed equally

Abbreviations: TNBC, triple negative breast cancer; NHERF1, $\mathrm{Na}^{+} / \mathrm{H}^{+}$exchanger regulatory factor 1 ; EGFR, epidermal growth factor receptor; coIP:mEGFR and coIP:mNHERF1, EGFR and NHERF1 coimmunoprecipitation of the membrane fraction respectively; coIP:eEGFR and coIP:eNHERF1, EGFR and NHERF1 coimmunoprecipitation of the endosome fraction respectively; WCL $\mathrm{mCL}$ eCL cCL whole, membrane, endosome and cytosolic cell lysates; WB, western blotting; TKI, tyrosine kinase inhibitor

Key words: NHERF1, EGFR, NHE1, breast cancer, triple negative, invadopodia, TKI inhibitors cell model, MDA-MB-231, and the role of forced NHERF1 overexpression and/or stimulation with EGF on the sensitivity to EGFR specific TKI treatment with gefitinib. Stimulation with EGF induces an interaction of NHERF1 with EGFR to regulate its localization, degradation and function. NHERF1 overexpression is sufficient to drive its interaction with EGFR in non-stimulated conditions, inhibits EGFR degradation and increases its retention time in the plasma membrane. Importantly, NHERF1 overexpression strongly sensitized the cell to the pharmacological inhibition by gefitinib of EGFR-driven growth, motility and invadopodia-dependent ECM proteolysis. The further determination of how the NHERF1-EGFR interaction is regulated may improve our understanding of TNBC resistance to the action of existing anticancer drugs.

\section{Introduction}

TNBC is a breast cancer subtype that is negative for estrogen and progesterone receptors and epidermal growth factor receptor 2 (HER2; ErbB2). TNBC accounts for approximately $15-20 \%$ of all breast cancer cases and seems to be closely related to basallike breast cancer (1). Patients with TNBC have a relatively poor outcome and cannot be treated with endocrine therapy or targeted therapies due to lack of related receptors (2). Thus, there is a substantial need for new therapies that can target TNBC and the progression of this disease. EGFR is a receptor tyrosine kinase whose function has been implicated in many biological processes; when activated, EGFR stimulates signalling pathways involved in cell growth, survival, and migration and its overexpression is the primary mechanism by which it contributes to breast cancer growth and progression (3). EGFR is overexpressed in TNBC; indeed, EGFR expression is one of the defining characteristics of TNBC and a predictor of poor prognosis (4). Several small molecule TKIs targeting EGFR have shown clinical efficacy in lung, pancreatic, colorectal, and head and neck cancers (5-8) and while phase II clinical studies have demonstrated that gefitinib (Iressa ${ }^{\circledR}$ ), in particular, shows antitumor activity in patients with other breast cancer types when used as a monotherapy or in combination with other drugs, such as docetaxel or anastrozole (9), little benefit 
has been noted in TNBC $(10,11)$ even though the EGFR is overexpressed (12-16).

Thus, a better comprehension of the downstream EGFR cellular events is required for the identification of molecular markers, which may allow the selection of patients more likely to benefit from treatment as well as for monitoring anti-EGFR therapies and indeed the development of novel treatment strategies for patients positive for EGFR but resistant to gefitinib and other anti-EGFR therapies.

In a metastatic basal like TNBC cell model, the downregulation of HIF-1 $\alpha$ through the EGFR signaling pathway appeared to be necessary for inducing a positive response to EGFR-targeted therapies and to gefitinib in particular, although it was demonstrated that this may not be sufficient (17). Indeed EGFR subcellular distribution to the nucleus, where it behaves as a transcription factor, has been implicated in enhancing proliferative potential and acquired resistance to gefitinib therapy (18).

Ligand-induced endocytosis and degradation of EGFR play important roles in the downregulation of the EGFR signal (19) suggesting that a way to regulate its activity could be to target its trafficking. The expression level of the scaffolding protein $\mathrm{Na}^{+} / \mathrm{H}^{+}$exchanger regulatory factor 1 (NHERF1) has been demonstrated to have profound effects on the trafficking, expression and function of the EGFR in normal cells $(20,21)$. NHERF1 is a 358-residue protein comprising two tandem PDZ domains (protein-binding domains conserved in the mammalian synaptic protein, PSD-95 Drosophila Dlg or discs large, and the adherens junction protein, $\mathrm{ZO}-1$ ) and a $\mathrm{COOH}$-terminal ERM binding region (22). In tumors, NHERF1 is overexpressed (23-26) and this is associated with a more aggressive behaviour and poor prognosis (27-29); its expression and subcellular localization can influence breast carcinogenesis $(30,31)$.

In non-tumor cells, EGFR binds to the PDZ1 domain of NHERF1 via an internal peptide motif located within the C-terminal regulatory domain of EGFR, thus slowing its degradation and enhancing its localization at the cell surface (21) and, in this way, modulates EGFR biological signalling function. Molecular alterations of the PDZ1 domain that abolish the recognition of EGFR sequence enhance the ligand-induced receptor downregulation. Interestingly, the same effect of EGFR downregulation can be achieved with a point mutation in the EGFR regulatory region or, at the transcriptional level, with polymorphisms in both the coding and regulatory regions (32). Indeed, NHERF1 can alter EGFR function via the formation of protein complexes around EGFR and NHERF1 has been shown to form a protein complex involving EGFR and NF2 tumor suppressor at the adherens-junctions and this interaction prevented EGFR from internalizing and signalling, clustering it in different parts of the plasma membrane by association with the actin cytoskeleton network (20).

Altogether, these data led us to hypothesize that NHERF1 expression levels regulate EGFR trafficking and functional expression in breast cancer cells and, in this way, modulate its role in cancer progression and cancer response to treatment. Here, we investigated, in the metastatic basal-like TNBC model, MDA-MB-231, the subcellular localization of NHERF1, its interaction with EGFR and the impact of NHERF1 overexpression on cancer cell sensitivity to anti-EGFR treatment.

\section{Materials and methods}

Cell culture. MDA-MB-231 cells were grown in Dulbecco's modified Eagle's medium high glucose $(4,500 \mathrm{mg} / \mathrm{l})$ supplemented with $\mathrm{NaHCO}_{3}(3,700 \mathrm{mg} / \mathrm{l}), 10 \%$ (v/v) heat-inactivated fetal bovine serum, L-glutamine $(2 \mathrm{mM})$, sodium-pyruvate $(1 \mathrm{mg} / \mathrm{ml})$ and penicillin $(100 \mathrm{U}) /$ streptomycin $(100 \mathrm{mg} / \mathrm{ml})$. Lines were grown in a $5 \% \mathrm{CO}_{2} / 95 \%$ air humidified incubator at $37^{\circ} \mathrm{C}$. For western blotting, coimmunoprecipitation (coIP) and immunofluorescence (IF) experiments, cells were deprived overnight and stimulated with EGF (50 ng/ml, Calbiochem, San Diego, CA, USA) for the indicated time.

Cells and expression vectors containing NHERF1 mutants. Expression vectors for wild-type (wt) NHERF1 and NHERF1 mutated in the PDZ1 domain (PDZ1mut) were developed as described $(33,34)$ and used for the transfection of the breast cancer MDA-MB-231 cell line. Cells transfected with $3 \mu \mathrm{g}$ of DNA construct in FuGENE6 transfection reagent (Roche, Milan, Italy) according to the manufacturer's protocol were maintained in complete medium containing $500 \mu \mathrm{g} / \mathrm{ml}$ hygromycin B (Calbiochem) and stable clones for wtNHERF1 and PDZ1mut expressing 3-fold NHERF1 levels and a pcDNA empty vector-expressing clone in which NHERF1 was expressed at endogenous levels were selected.

Western blotting. Samples were extracted in SDS sample buffer $(6.25 \mathrm{mM}$ Tris- $\mathrm{HCl}, \mathrm{pH} 6.8$, containing $10 \%$ glycerol, $3 \mathrm{mM}$ SDS, $1 \%$ 2-mercaptoethanol, and $0.75 \mathrm{mM}$ of bromophenol blue), separated by 4-12\% SDS-polyacrylamide gel electrophoresis and blotted to Immobilon P (Merck Millipore, Milan, Italy). Western blotting was performed with monoclonal antibodies (BD Biosciences Transduction Laboratories, Milan, Italy) against NHERF1 diluted 1:250 or EGFR diluted 1:250 against and diluted 1:2,500 against Actin (Sigma-Aldrich, Hamburg, Germany).

Cell fractionation. After treatment, monolayers were washed with PBS and then lysed in buffer A (10 mM HEPES, pH 7.9, $10 \mathrm{mM} \mathrm{KCl}, 0.1 \mathrm{mM}$ EDTA, $0.1 \mathrm{mM}$ EGTA, $1 \mathrm{mM}$ dithiothreitol and $0.5 \mathrm{mM}$ phenylmethylsulfonyl fluoride) and homogenized by five passes through a 20 -gauge needle to obtain the cell homogenate. An aliquot was removed for the determination of total cellular protein. The nuclear fraction was obtained by centrifuging the homogenate at $600 \mathrm{x} g$ for $10 \mathrm{~min}$. The resulting supernatant was centrifuged at $3500 \mathrm{x} \mathrm{g}$ for $10 \mathrm{~min}$ to obtain a pellet containing the endosomal fraction and the supernatant was centrifuged again at $17,000 \mathrm{x} \mathrm{g}$ for $1 \mathrm{~h}$ to obtain a plasma membrane pellet. The supernatant was centrifuged again at 100,000 x g for $1.5 \mathrm{~h}$, resulting in a microsomal pellet and the soluble cytoplasmic fraction in the supernatant. Thirty-five micrograms of each of the separated cellular fractions was extracted in SDS sample buffer and analyzed by western blotting.

Cross-linked gelatin layer preparation and fractionation. Cytosol, membrane and invadopodia fractions were obtained from cells grown on $2 \mathrm{mg} / \mathrm{ml}$ porcine skin gelatin in PBS containing $2 \mathrm{mg} / \mathrm{ml}$ sucrose as previously described (35). Briefly, $15 \mathrm{ml}$, kept warm at $40^{\circ} \mathrm{C}$, was spread on a $150-\mathrm{mm}$ 
diameter plastic dishes to evenly cover the entire dish surface. Excess gelatin was removed and the layer was maintained on ice for $10 \mathrm{~min}$. Then $10 \mathrm{ml}$ of ice cold, $0.5 \%$ glutaraldhyde in PBS was added for $15 \mathrm{~min}$ cross-link the gelatin. The cross-linked gelatin was then washed three times with PBS and $20 \mathrm{ml}$ of $70 \%$ ethanol was added to each dish for $1 \mathrm{~h}$ under a sterile bench hood to sterilize followed by two washes with sterile PBS for $5 \mathrm{~min}$ and two times with complete DMEM, the last wash of DMEM was not removed and the dishes were left in a humidfied, $37^{\circ} \mathrm{C}$ incubator for $1 \mathrm{~h}$ followed by seeding 4,000,000 cells on each dish, left for $24 \mathrm{~h}$ in a humidfied, $37^{\circ} \mathrm{C}, 5 \% \mathrm{CO}_{2}$ incubator. Cell fractions were isolated as follows: three washes with PBS containing $1 \mathrm{mM} \mathrm{CaCl}_{2}$ and $0.5 \mathrm{mM} \mathrm{MgCl}_{2}$, two times with $0.2 \mathrm{X}$ PBS plus $1 \mathrm{mM}$ $\mathrm{CaCl}_{2}$ and $0.5 \mathrm{mM} \mathrm{MgCl}$. Cells were then incubated on ice with $3 \mathrm{ml}$ of hypertonic swelling buffer [0.2X PBS supplied with $2 \mu \mathrm{l} / \mathrm{ml}$ protease inhibitor cocktail (Sigma-Aldrich), PMSF $1 \mathrm{mM}$, sodium orthovanadate $1 \mathrm{mM}$ ] for $15 \mathrm{~min}$ on ice. Cell bodies were gently scraped with an L-shaped pipette and centrifuged at $10,300 \times \mathrm{g}$ for $30 \mathrm{~min}$. Supernatant was collected (cytosolic soluble proteins) and placed on ice while the pellet was resuspended with $100 \mu 1$ of lysis buffer (HEPES $5 \mathrm{mM}$, EDTA $0.5 \mathrm{mM}$, pH 7.2 supplied with protease inhibitor $2 \mu \mathrm{l} / \mathrm{ml}$, PMSF $1 \mathrm{mM}$, sodium orthovanadate $1 \mathrm{mM}$, DTT $1 \mathrm{mM}$, nonidet $0.1 \%$ ) and membrane proteins were extracted by $30 \mathrm{~min}$ of rotating at $4^{\circ} \mathrm{C}$. After two PBS washes, the entire gelatin layer containing entrapped invadopodia was scraped from the dish with $1 \mathrm{ml}$ of the lysis buffer, vortexed and protein extracted for $30 \mathrm{~min}$ on an orbiting wheel at $4^{\circ} \mathrm{C}$. The fractions containing membrane proteins or invadopodia were collected in Eppendorf tubes and centrifuged at $13,000 \times \mathrm{rpm}$ at $4^{\circ} \mathrm{C}$. The supernatant of each fraction was collected and the pellet discarded. Protein concentration of the three fractions were measured with Bradford reagent (Pierce, Rockford, IL, USA). The diluted samples were precipitated with nine volumes of $-20^{\circ} \mathrm{C}$ acetone overnight and centrifuged at 10,300 x g for $1 \mathrm{~h}$. Proteins were resuspended in SDS sample buffer and western blotted.

Coimmunoprecipitation. After treatment monolayers were washed two times with ice-cold phosphate buffered saline (PBS), lysed in ice-cold coimmunoprecipitation lysis buffer (50 mM Tris, pH 7.5, $150 \mathrm{mM} \mathrm{NaCl,} \mathrm{1 \%} \mathrm{NP-40,} \mathrm{0.5 \%} \mathrm{sodium}$ deoxycholate, $100 \mathrm{mM} \mathrm{Na}_{3} \mathrm{VO}_{4}$ and $1 \mathrm{mM} \mathrm{NaF}$, protease inhibitors) and homogenated by five passes through a 20-gauge needle to obtain the total cell homogenate. An aliquot was removed for the determination of total cellular protein concentration of which $150 \mathrm{mg}$ was incubated for $1 \mathrm{~h}$ at $4^{\circ} \mathrm{C}$ on a rotator with primary antibody followed by the addition of protein A/G PlusAgarose (Santa Cruz Biotechnology, Santa Cruz, CA, USA) and incubation at $4^{\circ} \mathrm{C}$ overnight on a rotator. Immunoprecipitates were collected by centrifugation at $2,500 \mathrm{rpm}$ for $5 \mathrm{~min}$ at $4^{\circ} \mathrm{C}$. The pellet was washed four times with $1 \mathrm{ml}$ of lysis buffer and the pellet resuspended in $40 \mathrm{ml}$ of SDS sample buffer, run on $10 \%$ SDS-PAGE and analyzed by western blotting.

Immunofluorescence. Cells on coverslips were washed two times in sterile PBS at RT, fixed with $3.7 \%$ ice-cold paraformaldehyde/PBS for $20 \mathrm{~min}$, washed with ice-cold PBS, permeabilized with $0.1 \%$ Triton $\mathrm{X}-100$, saturated with $0.1 \%$ gelatin in PBS and then incubated with polyclonal antiNHERF1 primary antibody (Affinity Bio-Reagents, Golden, CO, USA) diluted 1:300 or monoclonal anti-EGFR primary antibody (BD Biosciences Transduction Laboratories) diluted 1:500 in $0.1 \%$ gelatin in PBS at RT for $1 \mathrm{~h}$. They were then washed with $0.1 \%$ gelatin in PBS and incubated at RT for $1 \mathrm{~h}$ with the Alexa 488 goat anti-rabbit secondary antibody or the Alexa 568 goat anti-mouse secondary antibody conjugate (Invitrogen, Carlsbad, CA, USA). The coverslips were washed with ice-cold PBS, mounted with Mowiol (Calbiochem) and observed on a BX40 microscope (Olympus, Tokyo, Japan) with a SenSys 1401E-Photometrics CCD camera (Roper Scientific, Tucson, AZ, USA).

MTT assay. gefitinib (Selleckchem, USA) was dissolved in DMSO to a final concentration of $0.01-100 \mu \mathrm{M}$, added at these concentrations to $1.5 \times 10^{3}$ cells in 96 -bottomed well plates and incubated for $72 \mathrm{~h}$. After the incubation, MTT (Sigma-Aldrich) was added at a concentration of $0.5 \mu \mathrm{g} / \mathrm{ml}$ to each well and incubated for $1 \mathrm{~h}$ in a humidified atmosphere, solubilized in $100 \mu \mathrm{l}$ DMSO for $2 \mathrm{~h}$ and absorbance was measured at 570 and $655 \mathrm{~nm}$ in a plate reader (Packard Spectra Count, Stanford, CA, USA). $\mathrm{IC}_{50}$ was calculated with CalculSyn software (Biosoft, Cambridge, UK).

Degradation assay. For combined localization of gelatinolytic activity and actin in the same section, in situ zymography using dye-quenched (DQ)-gelatin (Molecular Probes, Eugene, OR, USA) as a substrate for gelatinolytic activity, was performed followed by immunolocalization of actin as described (35).

Wound healing assay. Cells were seeded in 6-well culture dishes and grown to $80 \%$ confluence. Cells were then starved in DMEM overnight, and a wound was introduced with a micropipette tip. The wounded cells were washed to remove any suspended cells and further incubated in the presence of EGF with or without gefitinib. The plates were photographed at 0,24 and $48 \mathrm{~h}$ and the exact wound width was calculated by NIH Image J Software.

Statistical procedures. Student's t-test was applied to analyze the statistical significance between treatments and a $p<0.05$ was considered as significant. All comparisons were performed with InStat (GraphPad software, San Diego, CA, USA).

\section{Results}

NHERF1 is a molecular integrator of EGFR downstream events. To demonstrate the involvement of NHERF1 in EGFR signalling, we first performed fluorescent microscopic experiments with the metastatic basal like TNBC cell line, MDA-MB-231 (Fig. 1A). Cells were serum deprived overnight and stimulated with EGF $(100 \mathrm{ng} / \mathrm{ml})$ for $10 \mathrm{~min}$ and then immunostained with anti-EGFR and anti-NHERF1 antibodies. NHERF1 (green) did not co-localize with EGFR (red) in non-stimulated conditions (control), while a short exposure to EGF stimulated NHERF1 and EGFR co-localization (yellow arrow).

To biochemically validate this EGF-induced physical interaction of NHERF1 with EGFR, we performed coim- 
A
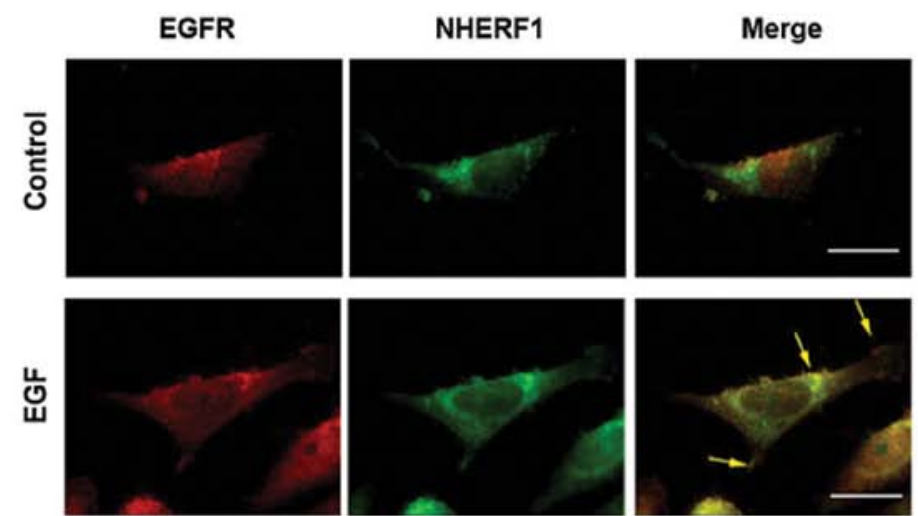

B

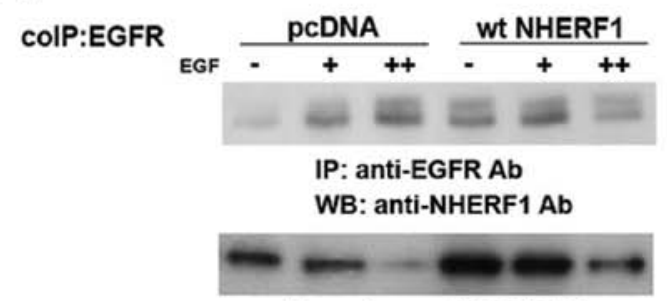

Reprobe : anti EGFR Ab
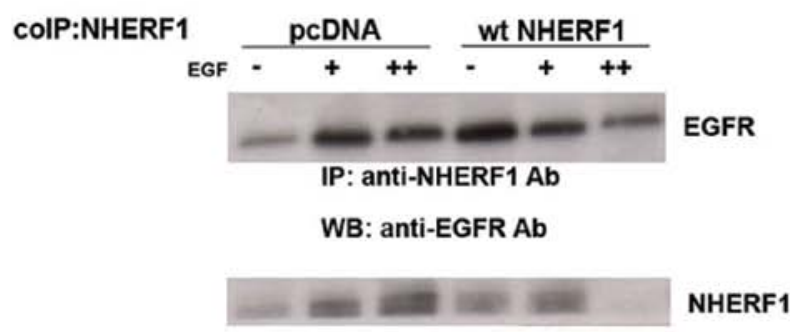

Reprobe : anti NHERF1 Ab

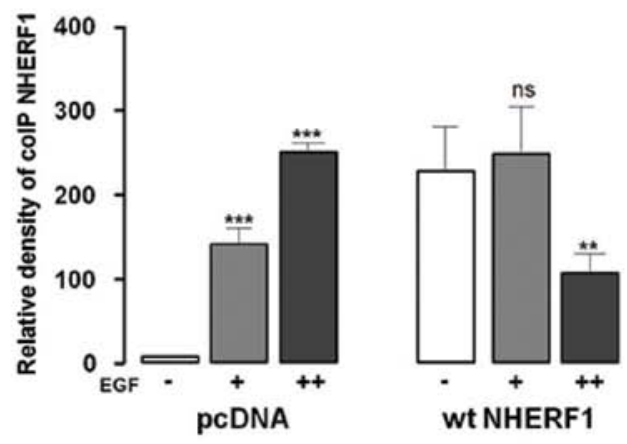

WCL $1 \%$

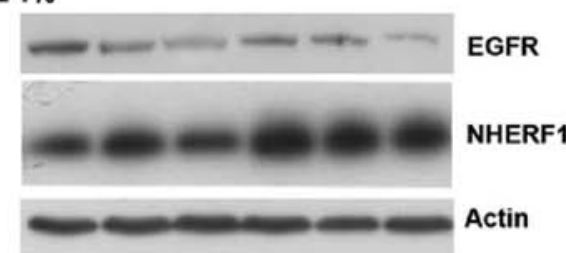

C
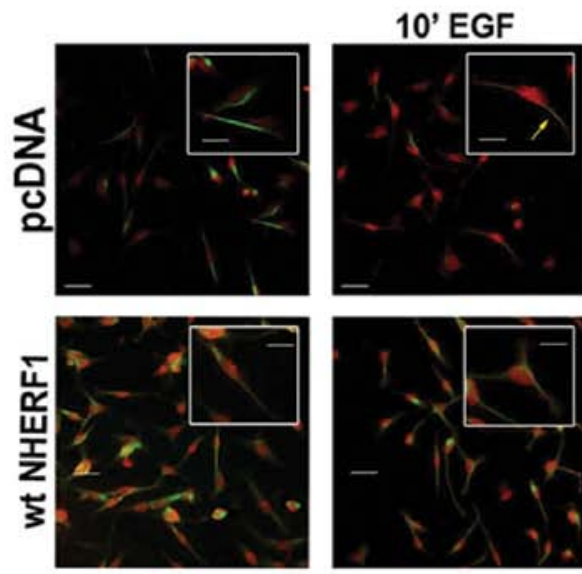

30' EGF
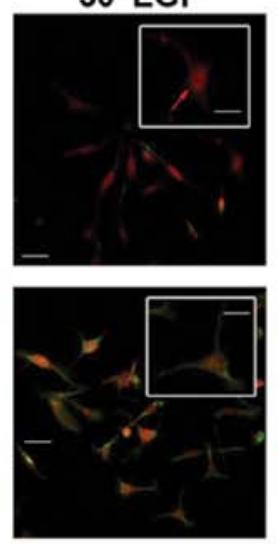

Figure 1. NHERF1 is involved in EGFR trafficking. (A) Representative MDA-MB-231 breast cells cultured and processed for immunofluorescence microscopy as described in Materials and methods. Cells were deprived overnight and stimulated with EGF (100 ng/ml) for $10 \mathrm{~min}$ and then immunostained with an antiEGFR mouse monoclonal antibody (red) and an anti-NHERF1 rabbit polyclonal antibody (green). Bars, $10 \mu \mathrm{m}$. (B) Reciprocal coIP of NHERF1 and EGFR. Left panel, representative coIP assays of the two proteins. Cells were stimulated with EGF and processed; EGFR was coIPed as described in Materials and methods. The empty plasmid transfected cells are indicated as pcDNA; cells transfected with the wild-type NHERF1 construct are indicated as wt NHERF1. Right panel shows the normalized quantification of coIPed complex expression performed using Image $J$ software. Results are represented as mean \pm SEM of four independent experiments performed. (C) Cells were treated and immunostained with an anti-EGFR mouse monoclonal antibody (green) and an antiNHERF1 rabbit polyclonal antibody (red) as described in Materials and methods. Bars, $10 \mu \mathrm{m}$ in the main image and $5 \mu \mathrm{m}$ in the insert. Unpaired Student's t-test ${ }^{* *} \mathrm{p}<0.01,{ }^{* * *} \mathrm{p}<0.001$; compared with the control.

munoprecipitation (coIP) experiments in cells stimulated with EGF for $10(+)$ or $30(++)$ min and, subsequently, immunoprecipitated with an anti-EGFR antibody (Fig. 1B). Levels of both endogenous EGFR and NHERF1 in the EGFR complex were measured by western blotting. In non-stimulated, empty plasmid transfected cells expressing normal endogenous levels 


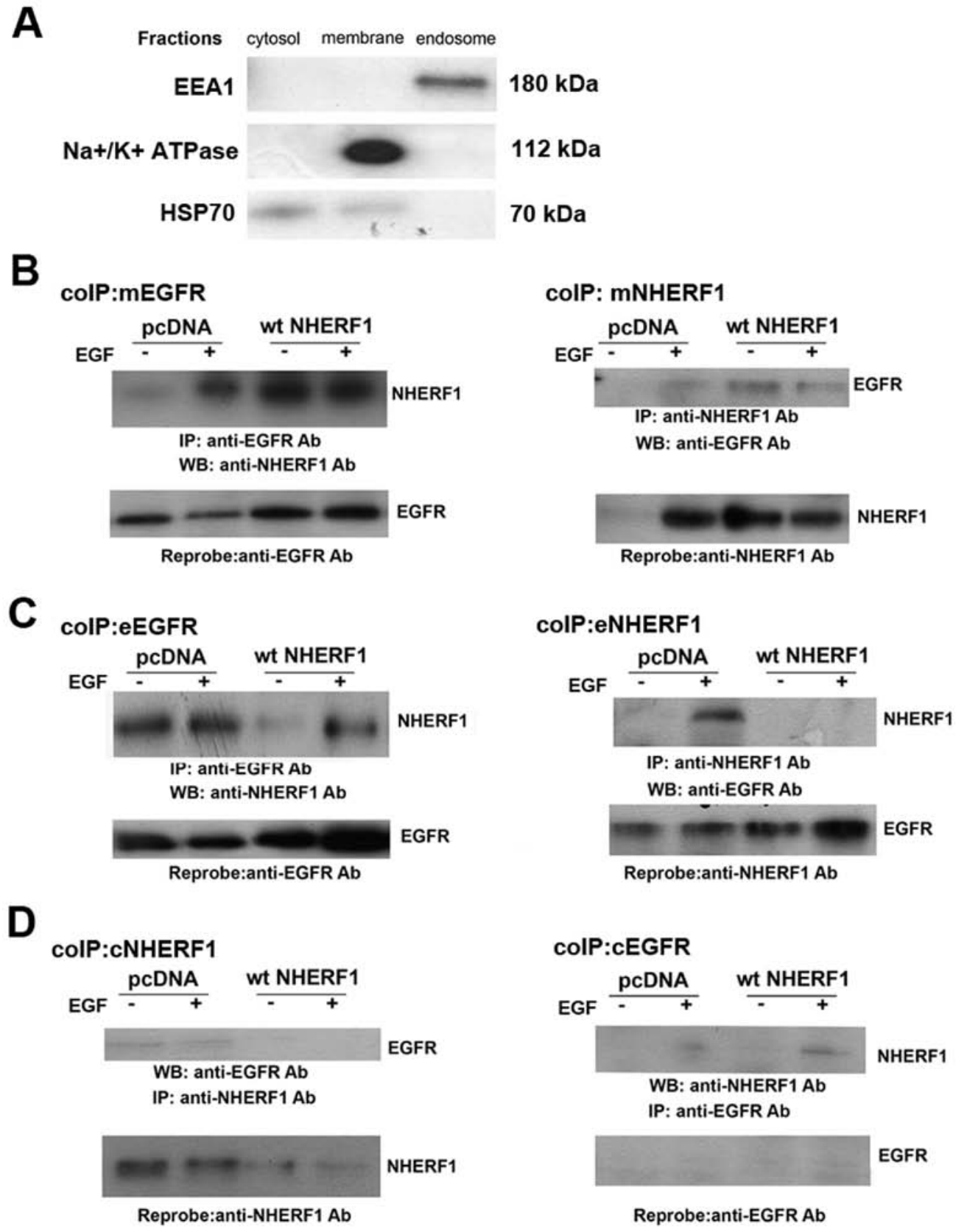

Figure 2. Study of interaction among EGFR and NHERF1 in tumor cell compartments. Cell treatment and fractionation was performed as described in Materials and methods. (A) Marker protein expression to determine purity of fractions. EEA1 (early endosome marker), $\mathrm{Na}^{+} / \mathrm{K}^{+}$-ATPase (membrane marker) and HSP70 (cytosol marker). (B) EGFR and NHERF1 reciprocally immunoprecipitated from membrane extract of MDA-MB-231 cells using first EGFR specific antibody and associated NHERF1 was detected by western blotting with NHERF1 specific antibody. (C) EGFR and NHERF1 were reciprocally immunoprecipitated from endosome extract of MDA-MB-231 cells. (D) EGFR and NHERF1 were reciprocally immunoprecipitated from cytosol extract of MDA-MB-231 cells.

of NHERF1 (pcDNA MDA-MB-231 cells), NHERF1 did not co-precipitate with EGFR and, upon EGF stimulation, the amount of co-precipitation of NHERF1 with EGFR increased with time. Conversely, in NHERF1 overexpressing cells (wtNHERF1), NHERF1 and EGFR already coIPed before EGF stimulation, and short term EGF stimulation transiently increased this phenomenon. Immunofluorescent studies confirmed these results (Fig. 1C). In pcDNA MDA-MB-231 cells (upper panels), NHERF1 (red) did not co-localize with EGFR (green) in non-stimulated conditions, while after a short time exposure to EGF, NHERF1 and EGFR co-localized in the plasma membrane (yellow arrow). Forced ectopic overexpression of NHERF1 (lower panels) resulted in the co-localization of the two proteins at the plasma membrane already in nonstimulated conditions and, after short EGF exposure, the main fraction of the EGFR-NHERF1 pair remained in the plasma membrane with only a much smaller portion also being observed in the perinuclear compartment.

To better understand the dynamics of interaction between NHERF1 andEGFR, we performed reciprocal NHERF1/EGFR coIP experiments of plasma membrane, endosome and cytosol (cL) cell fractions (Fig. 2). In the plasma membrane fraction 

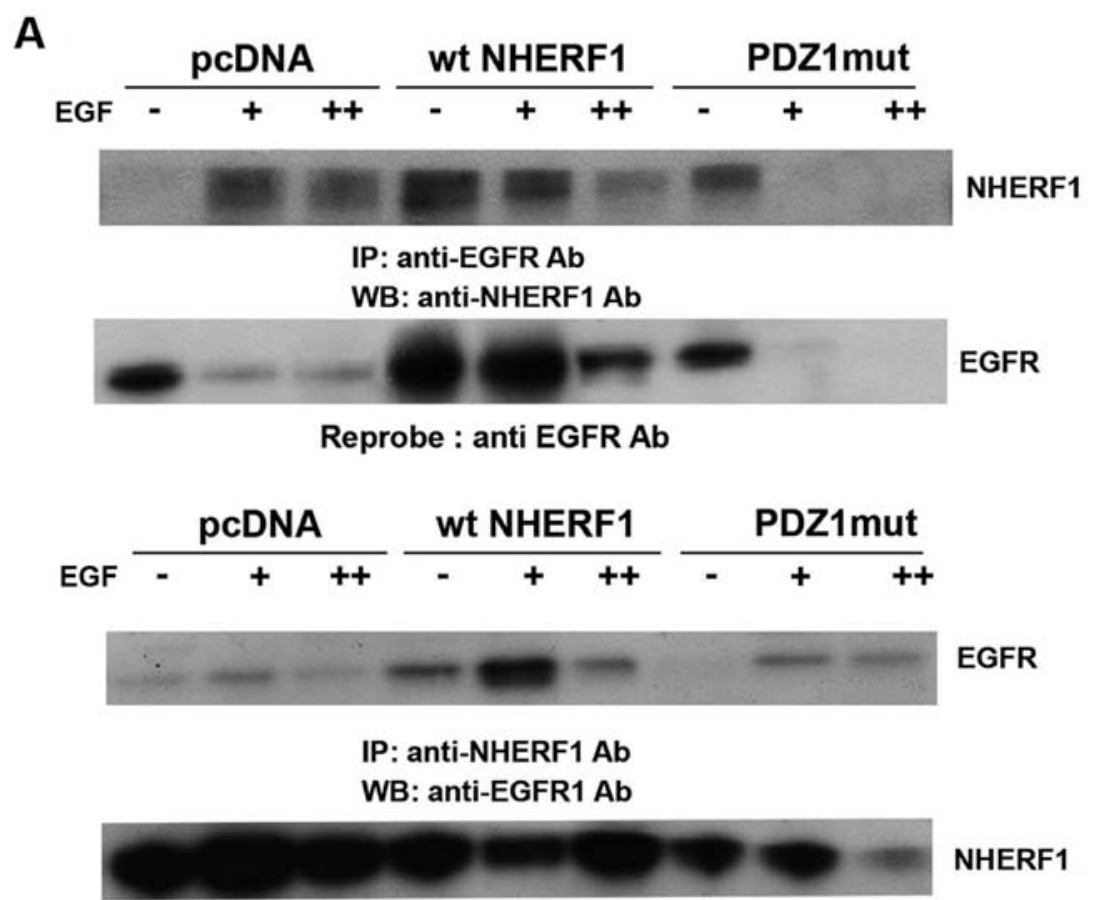

NHERF1

B

Reprobe : anti NHERF1 Ab

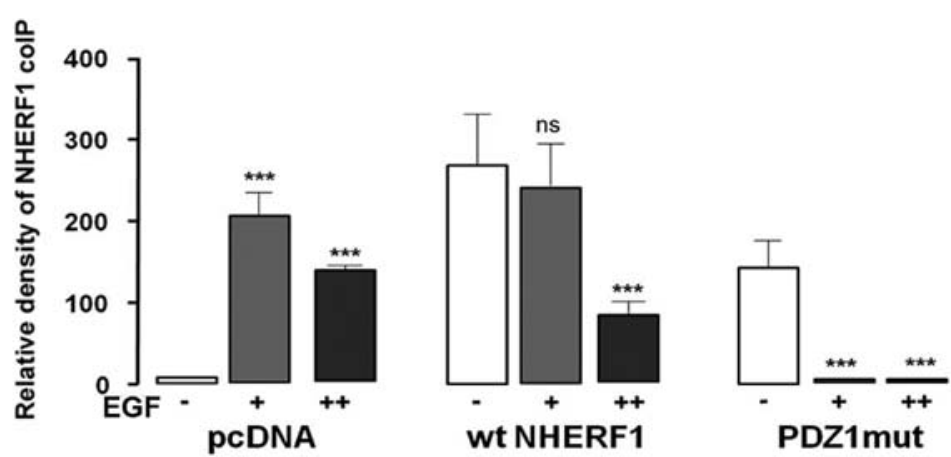

Figure 3. EGFR interacts with NHERF1 via its PDZ1 domain. MDA-MB-231 cells were stimulated with 100 ng/ml EGF for 10 (+) or 30 min (++) and EGFR was coIPed as described in Materials and methods. (A) Representative coIP assay of NHERF1 and EGFR in the membrane fraction. Cells were treated and the membrane fraction was isolated as described in Materials and methods. (B) Normalized quantification of expression performed using Image $\mathbf{J}$ software. Results are represented as mean \pm SEM of four independent experiments. Unpaired Student's t-test ${ }^{* * *}$ p $<0.001$; compared with the control.

of pcDNA MDA-MB-231 cells (Fig. 2A), EGF stimulation increased coIPed NHERF1 and decreased EGFR expression. When NHERF1 was overexpressed, NHERF1 always coIPed with the receptor and EGFR levels were not downregulated by EGF stimulation. In the endosomal fraction (Fig. 2B), we observed that EGF stimulation decrease the receptor level in the pcDNA cells, while the overexpression of NHERF1 both increased the amount of EGFR and decreased its degradation in the early endosome fraction. NHERF1 overexpression firstly completely abrogated their interaction in non-stimulated conditions restoring the complex after the EGF stimulation. The EGFR was never expressed in the cytosolic fraction (cCL) (Fig. 2C) of either pcDNA or wtNHERF1 transfected cells and in NHERF1 overexpressing cells, NHERF1 decreased with stimulation.

Altogether, these results demonstrate that EGF treatment in breast cells recruits NHERF1 from the cytosol to the plasma membrane, forming a complex with EGFR. On the contrary, the overexpression of NHERF1 results in an increased recycling of EGFR back to and an increased stability at the plasma membrane together with an increase of the NHERF1/EGFR complex and a reduction of this complex in the endosomes.

The PDZ1 domain of NHERF1 binds with EGFR. It has been hypothesized that in non-tumor cells EGFR binds the PDZ1 domain of NHERF1 (21) and we verified this hypothesis, in our in vitro model, with coIP experiments of the membrane fraction using cells transfected with PDZ1 mutated NHERF1 which can no longer bind its protein partners (PDZ1mut). As shown in Fig. 3A, forced ectopic wild-type (wt) NHERF1 overexpression increased NHERF1 coIPed with EGFR while overexpressing PDZ1mut NHERF1 strongly reduced its co-precipitation with EGFR. Moreover, overexpressing wt NHERF1 downregulated the rate of EGFR decrease after EGF treatment, while, when the PDZ1 domain function was lost (ectopic PDZ1mut), NHERF1 overexpression was no 
A TKI $\frac{\text { pcDNA }}{-} \quad \frac{\text { wt NHERF1 }}{-} \quad \frac{\text { PDZ1mut }}{-}$
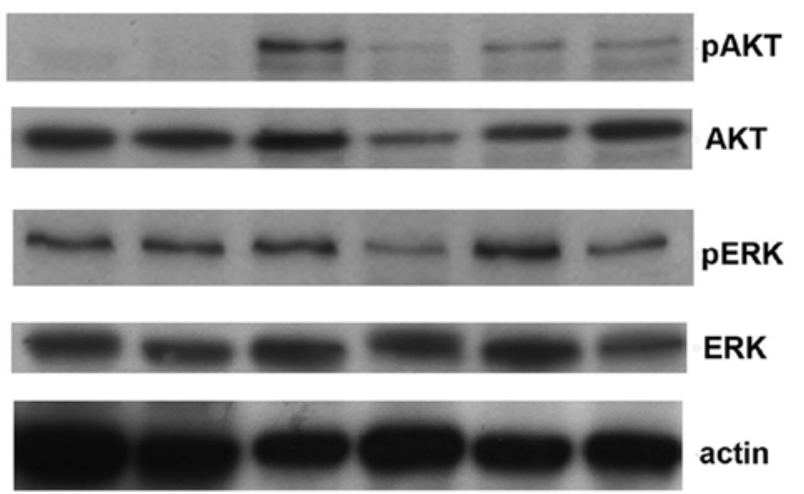

B

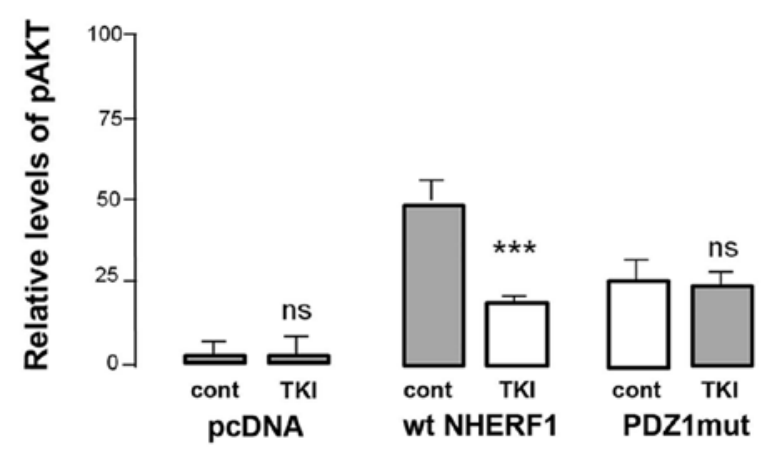

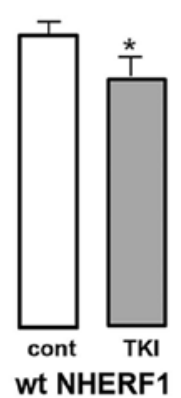

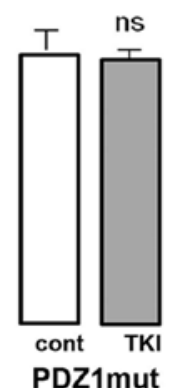

C

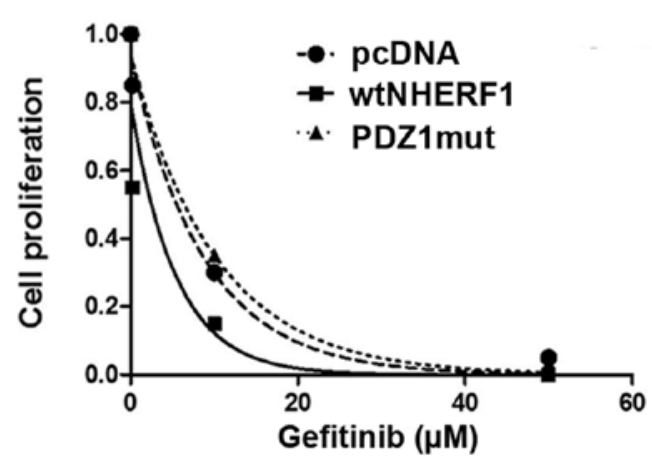

Figure 4. Effect of TKI gefitinib on tumor cell signalling pathways and growth. (A) Detection of AKT, pAKT, ERK and pERK in TNBC cells treated with gefitinib for $24 \mathrm{~h}$. Representative data from two independent experiments are shown. Cells were harvested and cell lysate were analysed by western blotting with the indicated antibodies. $\beta$-actin was used as a loading control. (B) Relative levels of pAKT and pERK. P-values were calculated for each treatment compared with untreated cells, using a two-tailed t-test. (C) MDA-MB-231 pcDNA and wtNHERF1 were assayed for studying the relevance of NHERF1 expression and interaction on the determination of long-term growth response to gefitinib. Cells were treated as described in Materials and methods. The kinetics of the growth curves were calculated with CalculSyn software. Results are represented as mean \pm SEM of four independent experiments performed in triplicate. Unpaired Student's t-test ${ }^{*} \mathrm{p}<0.05,{ }^{* * *} \mathrm{p}<0.001 ;$ compared with the control. longer able to prevent EGFR decrease (Fig. 3B). Overall, these data agree with Lazar et al (21) that EGFR and NHERF1 interact through the PDZ1 domain of NHERF1, also in breast cancer cells.

Relevance of NHERF1 expression levels in anti-EGFR drug activity. Alterations in receptor expression and localization have been shown to influence their reactivity to both ligands and to inhibitors. Since EGFR inhibition is an important antitumor therapeutic strategy we investigated the role of NHERF1 expression in the action of one of the well known EGFR inhibitors actually in clinical practice for anticancer therapy, the EGFR tyrosine kinase inhibitor (TKI), gefitinib (Iressa).

We first examined the effect of gefitinib on signaling pathways by a series of western blot analyses (Fig. 4A) and observed that the basic levels of phospho-AKT (pAKT) were low while significant levels of phospho-ERK (pERK) were detected. Treatment with gefitinib had no effect on pAKT or pERK levels in either pcDNA or PDZ1mut cells, while the level decreased in wt-NHERF1 cells (Fig. 4B). These data indicate that overexpression of NHERF1 may be required to render cells sensitive to gefitinib. Indeed, analyzing the doseresponse of gefitinib inhibition of cell growth of the three cell lines (Fig. 4C), only overexpression of wtNHERF1 sensitized this resistant cell to anti-EGFR therapy with gefitinib (shift of the $\mathrm{IC}_{50}$ from $6.36 \pm 0.52$ to $2.34 \pm 0.17 \mu \mathrm{M}$ ).

However, the highest levels of cancer morbidity depends on invasion and metastasis rather than growth (36) and the EGFR is known to be involved in tumor cell invasion through an increase in both cell migration and invadopodia-dependent digestion of the extracellular matrix (ECM) (35-37). These important processes have been rarely measured in studies on the effects of blocking EGFR activity on tumor phenotypes. For this reason, we measured the effect of NHERF1 overexpression on the effectiveness of anti-EGFR therapy directed against migration and against invadopodia-dependent ECM digestion.

Wound healing measurements utilised to test the ability of gefitinib to modulate MDA-MB-231 migration (Fig. 5) revealed that control monolayers displayed rapid wound healing within $48 \mathrm{~h}$ that was blocked $\sim 50 \%$ by treatment with gifitinib while PDZ1mut overexpression abrogated the ability of gefitinib to block motility. Importantly, overexpression of wtNHERF1 per se slightly reduced motility in the untreated cells but greatly sensitized the monolayers to inhibition of motility by gefitinib, especially at $48 \mathrm{~h}$ (an $\sim 75 \%$ reduction in motility).

We next measured the ability of MDA-MB-231 cells to form invadopodia and digest the ECM. To first obtain a quantitative comparison of the distribution of EGFR and the $\mathrm{Na}^{+} / \mathrm{H}^{+}$exchanger isoform 1 (NHE1) in invadopodia in the different clones and the effect of EGF treatment in pcDNA cells, cells were plated on $2 \%$ cross-linked gelatin, fractionated for cytosol, cell membrane and invadopodia fractions as previously described (35) and assayed by western blotting. As can be seen in Fig. 6A, overexpression of wtNHERF1 but not PDZ1mut-NHERF1 shifted a major fraction of the EGFR and NHE1 to the invadopodia. Cortactin was utilized as a control protein for the purity of the fractions (35). Interestingly, treatment of pcDNA cells with $50 \mathrm{ng} / \mathrm{ml}$ of EGF resulted in an 

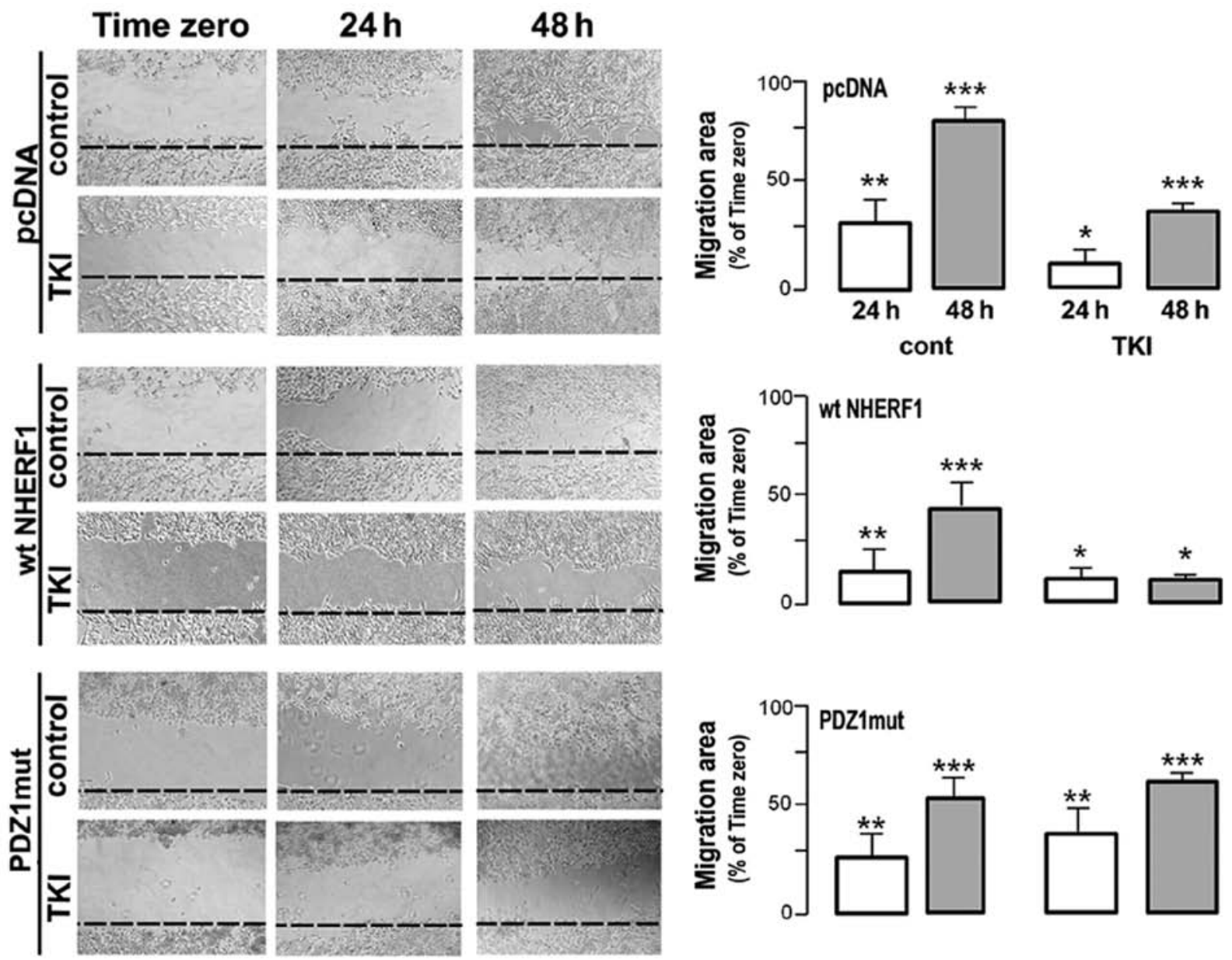

Figure 5. NHERF1 expression alters the effect of gefitinib on tumor motility. A wound healing assay utilized to study modulation on cell migration of the TKI, gefitinib, in MDA-MB-231 pcDNA, wtNHERF1 and PDZmut cells. Cells were treated as described in Materials and methods. The lower panel shows the migration measured using Image $\mathbf{J}$ software and normalized as the percent of time zero. Results are represented as mean \pm SEM of three independent experiments performed in duplicate. Unpaired Student's t-test ${ }^{*} \mathrm{p}<0.05,{ }^{* *} \mathrm{p}<0.01,{ }^{* * *} \mathrm{p}<0.001 ;$ compared with the control.

increase in p-EGFR in both the membrane and invadopodia fractions and a strong increase in NHE1 expression in the invadopodia (Fig. 6B).

We then measured invadopodia activity by microscopically measuring the release of quenched Bodipy fluorescence after 6-h incubation on 3D lattices of Matrigel-DQ-gelatin as previously described (35). After 6-h incubation on MatrigelDQ-gelatin, the cells formed bright F-actin puncta associated with focal matrix degradation, demonstrating functional invadopodia formation. Measuring the proteolytic ability of the cells via the intensity of the digestive fluorescent signal (Fig. 6C, left panel), revealed that wtNHERF1 overexpression greatly enhanced invadopodia-dependent ECM digestion while PDZ1mut overexpression had no effect on the proteolytic activity of invadopodia. Importantly, gefitinib treatment downregulated invadopodia-dependent matrix degradation process by $\sim 50 \%$ in pcDNA cells and by $80 \%$ in wtNHERF1 overexpressing cells. Performing these experiments in the presence or absence of EGF and or $1 \mu \mathrm{M}$ of the specific NHE1 inhibitor, cariporide, demonstrated that $\sim 50 \%$ of basal ECM proteolysis and $65-75 \%$ of that stimulated by either wtNHERF1 transfection or $50 \mathrm{ng} / \mathrm{ml}$ EGF treatment was dependent on NHE1 activity (Fig. 6C, right panel). Altogether, these data show that NHERF1 acts primarily at the level of the invadopo- dial digestive ability, and that treatment with gefitinib or with cariporide blocks invadopodia function. Finally, wtNHERF1 overexpression rendered the cells more sensitive to inhibition of ECM digestion with gefitinib.

\section{Discussion}

The roles of EGFR in carcinogenesis are well known and its signalling pathway is presently an attractive target for therapy in a large number of tumor types $(38,39)$. Ligand-induced endocytosis and degradation of EGFR play important roles in the downregulation of the EGFR signal (19) and, consequently, in both the expression of neoplastic phenotypes and in the dynamics of inhibitor action. In normal cells, the level of NHERF1 expression has been demonstrated to have profound effects on the trafficking, expression and function of the EGFR (20).

Here we find that, also in breast cancer cells, NHERF1 is an important molecular integrator of EGFR trafficking and that this regulation is important in determining the cells aggressive behaviour and its response to anti-EGFR therapy. The immunofluorescence and co-immunoprecipitation studies demonstrated that EGFR stimulation in response to EGF drove the relocalization of cytosolic NHERF1 first to the 

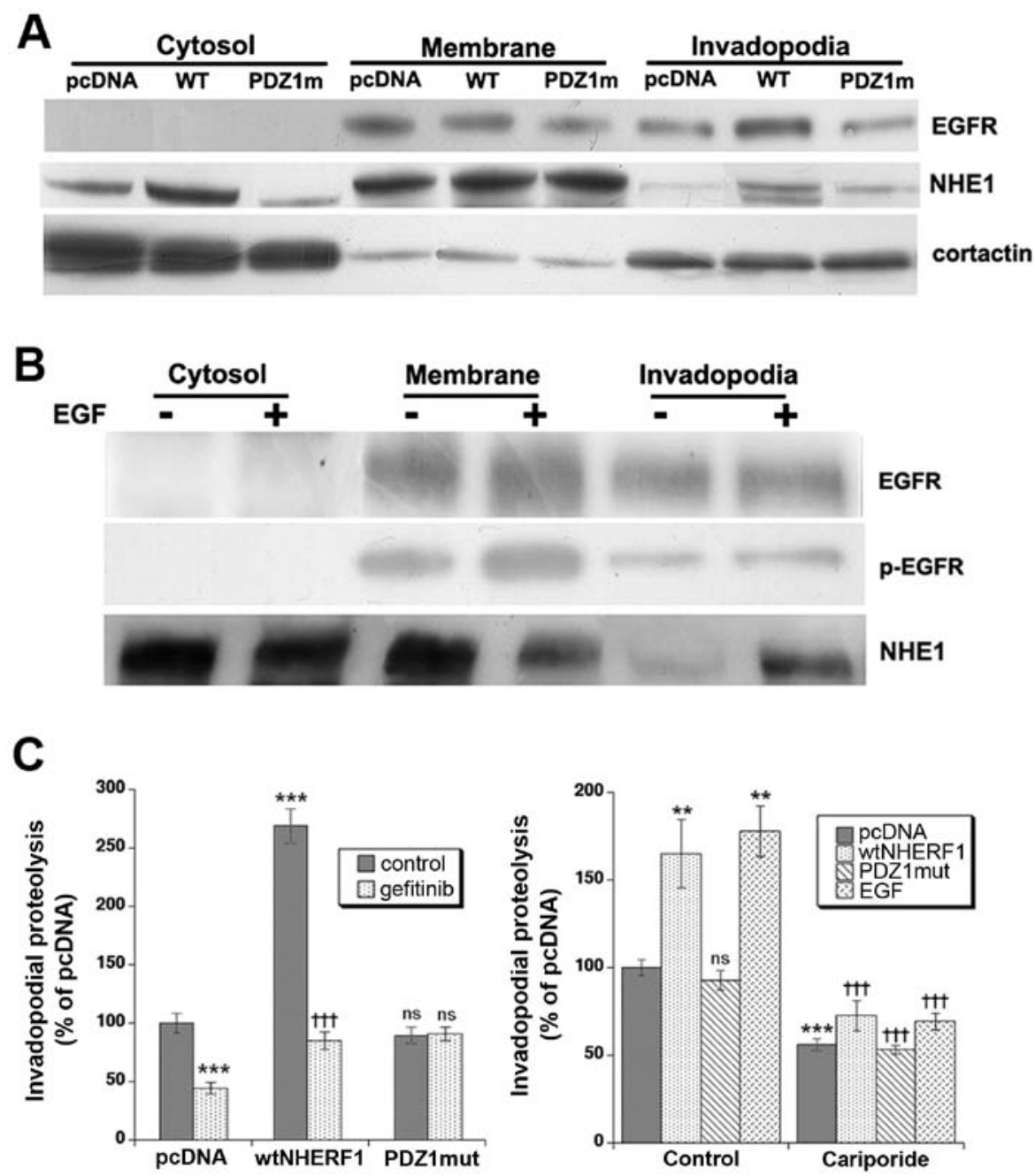

Figure 6. NHERF1 expression alters gefitinib effect on invadopodia expression and proteolytic capacity. Fractions were isolated from cells plated on gelatin and fractionated as per Materials and methods. (A) Left upper panel, western blotting of EGFR, NHE1 and cortactin (invadopodia enrichment marker) in fractions from cells transfected with the empty vector (pcDNA), wild-type NHERF1 (wtNHERF1) or NHERF1 mutated in the PDZ1 domain (PDZ1mut) such that it no longer binds protein partners. The cytosol does not contain EGFR. (B) Western blot analysis of EGFR and p-EGFR and NHE1 in fractions from pcDNA cells before and after $50 \mathrm{ng} / \mathrm{ml}$ EGF treatment for $6 \mathrm{~h}$. (C) DQ-gelatin degradation assay was used to analyse the proteolytic activity of invadopodia in F-actin focal points on the degradated Matrigel matrix. Left panel shows the analysis of the effect of NHERF1 expression modulation and gefitinib treatment on the invadopodia proteolytic activity. Right panel shows the effect of $1 \mu \mathrm{M}$ cariporide on invadopodia-dependent degradation of Matrigel matrix in cells transfected with wtNHERF1,PDZ1mut or after $50 \mathrm{ng} / \mathrm{ml}$ EGF. Values are expressed as mean \pm SEM of five independent experiments. Unpaired Student's t-test ${ }^{* *} \mathrm{p}<0.01,{ }^{* * * *} \mathrm{p}<0.001$; compared with the control and ${ }^{i \dagger} \mathrm{p}<0.001$ of gefitinib compared to its respective control or cariporide for each treatment compared to its respective control.

plasma membrane compartment followed by a co-transport to the perinuclear region. Moreover, we observed that, upon EGF stimulation, NHERF1 colocalized with EGFR and our data suggest that EGF treatment first downregulates EGFR expression probably through the internalization and degradation of the receptor followed by, when stimulated for longer times, its trafficking to other cell compartments as the nucleus (40). Ectopic NHERF1 overexpression reduced EGFR degradation (Fig. 1B) and increased its expression at the plasma membrane (Fig. 2B). This increased plasma membrane expression linked to reduced degradation when NHERF1 was overexpressed led us to determine if NHERF1 stabilizes EGFR at the membrane, as previous described (21), or if there is more EGFR at the plasma membrane through a modulation of its recycling to the membrane. Therefore, we measured the influence of NHERF1 expression levels and EGF stimulation on the dynamics of the EGFR/NHERF1 complex by analyzing their expression levels and interaction in cell fractionation/coIP experiments
(Fig. 2). These experiments showed that in cells expressing endogenous NHERF1, EGF stimulation recruits endogenous NHERF1 from the cytosol to the plasma membrane forming a complex with EGFR that drives degradation of the receptor. When NHERF1 is overexpressed, ectopic NHERF1 already complexes with EGFR in non-stimulated conditions and increases the EGFR plasma membrane residence time in EGF stimulation, through an increased recycling of the EGFR from the endosome fraction back to the plasma membrane. Altogether, these data suggest that the cellular NHERF1 expression level functions to shift the cellular equilibrium from one EGFR localization and regulatory cascade/scenario to another. The mechanism by which the breast cancer cell can alter NHERF1 expression levels and, consequently shift the balance of recycling endosomes, is suggested by data showing that exposure of the cancer cell to hypoxia or low nutrients increases NHERF1 expression and localization resulting in an increase in invasion (26). 
Indeed, this increased retention time (i.e., more stable expression) of the EGFR in the plasma membrane and the reduction of the negative control the EGFR signalling cascade should increase the cells aggressiveness. However, it could, possibly, also increase its sensitivity to anti-EGFR phosphotyrosine kinase inhibitor therapy. To test this hypothesis, we next analysed the relevance of NHERF1 expression on the effect of response to the tyrosine kinase inhibitor (TKI), gefitinib (Iressa) on signalling pathways downstream the EGFR activation (Fig. 4A) and we observed that the overexpression of NHERF1 and its PDZ1 mutation is able per se to increase the basal level of phosphor-AKT (Fig. 4B), but gefitinib downregulated the AKT activation only with a functional PDZ1 domain. Gefitinib is also able to slightly regulate the activation of ERK. We also analysed the effect of NHERF1 expression on the effect of gefitinib on growth and we observed that, indeed, its sensitized the cell lines to gefitinib (Fig. 4C), demonstrating that the TNBC cell endogenous levels of NHERF1 could be functional but not sufficiently available for an enhanced gefitinib efficacy. As the highest levels of cancer morbidity depend on invasion and metastasis, we next determined the role of NHERF1 expression levels also on motility, invasion and proteolysis of the extracellular matrix (ECM). The wound healing motility test (Fig. 5) revealed that, indeed, NHERF1 overexpression sensitized the monolayer to gefitinib treatmentdependent inhibition of in vitro cell migration.

We next analysed in detail the presence and proteolytic activity of invadopodia (Fig. 6) and observed that ectopic NHERF1 overexpression, as hypothesized, i) increased invadopodia formation and the degradation of the extracellular matrix; and ii) strongly increased the inhibitory effect of gefitinib on both the formation of invadopodia and, more strongly, on their proteolytic activity. These data are interesting in the context of a previous paper showing that activation of the EGFR can initiate invadopodia maturation via the subsequent activation of a Src-Arg-cortactin pathway that organizes the recruitment of Arp 2/3, Nck 1 and N-WASp proteins to the local cytoskeleton (37). Our data suggest that although the EGFR can act to initiate invadopodia formation, its primary action is to stimulate/regulate invadopodia-dependent ECM proteolysis. Our observation that gefitinib had a very high capacity to block the NHERF1-dependent increase in proteolytic activity suggests that the EGFR, when stabilized at the membrane by NHERF1, may act primarily at the level of invadopodia proteolytic activity. This may probably occur through an activation of the NHE1 as it has been demonstrated that both the overexpression of NHERF1 (26) and stimulation by EGF (35) drive an enhanced extracellular acidification and, consequently, an enhanced protease activity by activating the NHE1 (41). Indeed, both EGF treatment and overexpression of wt-NHERF1, but not PDZ1mut NHERF1, increased the level of NHE1 in the invadopodia and the specific NHE1 inhibitor, cariporide, reduced both basal and EGF-stimulated invadopodia-driven focal ECM proteolysis (Fig. 6).

In conclusion, a number of mechanisms have been suggested for resistance to EGFR TKI-induced growth inhibition in cancers, including EGFR independence, mutations in EGFR and alterations in downstream signalling pathways. Here, we show that the expression level of the signal transduction scaffolding protein NHERF1, regulates EGFR recycling/ degradation to stabilize the EGFR on the plasma membrane and sensitize the cell to the TKI-dependent inhibition of EGFR-driven motility and invadopodia-dependent ECM proteolysis in cancer cells. The identification of the NHERF1EGFR network and the determination of how it is regulated may improve our understanding of the cancer metastasis process, and the optimization of current anticancer drugs specifically targeting this process.

\section{Acknowledgements}

We thank Professor E. Weinman (Department of Medicine, University of Maryland School of Medicine, Baltimore, MD, USA) for the gift of the NHERF1 wild-type and PDZ1 domain mutated (PDZ1MUT) constructs. S.J.R. would like to thank the Italian Association for Cancer Research (AIRC) grant no. 11348 for supporting this study. K.Z. is a fellow of the Marie Curie Initial Training Network IonTraC (FP7-PEOPLE2011-ITN Grant Agreement no. 289648). The S.J.R. laboratory is part of the Italian network 'Istituto Nazionale Biostrutture e Biosistemi' (INBB) and the 'Centro di Eccellenza di Genomica in Campo Biomedico ed Agrario' of the University of Bari and the project BioBoP of the region Puglia.

\section{References}

1. Foulkes WD, Smith IE and Reis-Filho JS: Triple-negative breast cancer. N Engl J Med 363: 1938-1948, 2010.

2. Metzger-Filho O, Tutt A, de Azambuja E, Saini KS, Viale G, Loi S, et al: Dissecting the heterogeneity of triple-negative breast cancer. J Clin Oncol 30: 1879-1887, 2012.

3. Paez JG, Janne PA, Lee JC, Tracy S, Greulich H, Gabriel S, et al: EGFR mutations in lung cancer: correlation with clinical response to gefitinib therapy. Science 304: 1497-1500, 2004.

4. Korsching E, Jeffrey SS, Meinerz W, Decker T, Boecker W and Buerger H: Basal carcinoma of the breast revisited: an old entity with new interpretations. J Clin Pathol 61: 553-560, 2008.

5. Baker M: EGFR inhibitors square off at ASCO. Nat Biotechnol 22: 641, 2004.

6. Cohen EE, Lingen MW, Martin LE, Harris PL, Brannigan BW, Haserlat SM, et al: Response of some head and neck cancers to epidermal growth factor receptor tyrosine kinase inhibitors may be linked to mutation of ERBB2 rather than EGFR. Clin Cancer Res 11: 8105-8108, 2005.

7. Giusti RM, Shastri K, Pilaro AM, Fuchs C, Cordoba-Rodriguez R, Koti K, et al: U.S. Food and Drug Administration approval: panitumumab for epidermal growth factor receptor-expressing metastatic colorectal carcinoma with progression following fluoropyrimidine-, oxaliplatin-, and irinotecan-containing chemotherapy regimens. Clin Cancer Res 14: 1296-1302, 2008.

8. Sobrero AF, Maurel J, Fehrenbacher L, Scheithauer W, Abubakr YA, Lutz MP, et al: EPIC: phase III trial of cetuximab plus irinotecan after fluoropyrimidine and oxaliplatin failure in patients with metastatic colorectal cancer. J Clin Oncol 26: 2311-2319, 2008.

9. Polychronis A, Sinnett HD, Hadjiminas D, Singhal H, Mansi JL, Shivapatham D, et al: Preoperative gefitinib versus gefitinib and anastrozole in postmenopausal patients with oestrogen-receptor positive and epidermal-growth-factor-receptor-positive primary breast cancer: a double-blind placebo-controlled phase II randomised trial. Lancet Oncol 6: 383-391, 2005.

10. Johnston JB, Navaratnam S, Pitz MW, Maniate JM, Wiechec E, Baust H, et al: Targeting the EGFR pathway for cancer therapy. Curr Med Chem 13: 3483-3492, 2006.

11. Montemurro F, Valabrega G and Aglietta M: Lapatinib: a dual inhibitor of EGFR and HER 2 tyrosine kinase activity. Expert Opin Biol Ther 7: 257-268, 2007.

12. Baselga J, Albanell J, Ruiz A, Lluch A, Gascón P, Guillém V, et al: Phase II and tumor pharmacodynamic study of gefitinib in patients with advanced breast cancer. J Clin Oncol 23: 5323-5333, 2005. 
13. Tan AR, Yang X, Hewitt SM, Berman A, Lepper ER, Sparreboom A, et al: Evaluation of biologic end points and pharmacokinetics in patients with metastatic breast cancer after treatment with erlotinib, an epidermal growth factor receptor tyrosine kinase inhibitor. J Clin Oncol 22: 3080-3090, 2004.

14. Irwin ME, Mueller KL, Bohin N, Ge Y and Boerner JL: Lipid raft localization of EGFR alters the response of cancer cells to the EGFR tyrosine kinase inhibitor gefitinib. J Cell Physiol 226 2316-2328, 2011

15. Metro G, Finocchiaro G and Cappuzzo F: Anti-cancer therapy with EGFR inhibitors: factors of prognostic and predictive significance. Ann Oncol (Suppl 2): ii42-ii45, 2006.

16. She QB, Solit D, Basso A and Moasser MM: Resistance to gefitinib in PTEN-null HER-overexpressing tumor cells can be overcome through restoration of PTEN function or pharmacologic modulation of constitutive phosphatidylinosito 3'-kinase/Akt pathway signaling. Clin Cancer Res 9: 43404346, 2003.

17. El Guerrab A, Zegrour R, Nemlin CC, Vigier F, Cayre A, Penault-Llorca F, et al: Differential impact of EGFR-targeted therapies on hypoxia responses: implications for treatment sensitivity in triple-negative metastatic breast cancer. PLoS One 6: e25080, 2011.

18. Yu YL, Chou RH, Liang JH, Chang WJ, Su KJ, Tseng YJ, et al: Targeting the EGFR/PCNA signaling suppresses tumor growth of triple-negative breast cancer cells with cell-penetrating PCNA peptides. PLoS One 8: e61362, 2013.

19. Yarden Y and Sliwkowski MX: Untangling the ErbB signalling network. Nat Rev Mol Cell Biol 2: 127-137, 2001.

20. Curto M, Cole BK, Lallemand D, Liu CH and McClatchey AI: Contact dependent inhibition of EGFR signaling by Nf2/Merlin. J Cell Biol 177: 893-903, 2007.

21. Lazar CS, Cresson CM, Lauffenburger DA and Gill GN: The $\mathrm{Na}^{+} / \mathrm{H}^{+}$exchanger regulatory factor stabilizes epidermal growth factor receptors at the cell surface. Mol Biol Cell 15: 5470-5480, 2004.

22. Bretscher A, Chambers D, Nguyen R and Reczek D: ERM-Merlin and EBP50 protein families in plasma membrane organization and function. Annu Rev Cell Dev Biol 16: 113-143, 2000.

23. Shibata T, Chuma M, Kokubu A, Sakamoto M and Hirohashi $S$ EBP50, a beta-catenin-associating protein, enhances Wnt signaling and is over-expressed in hepatocellular carcinoma. Hepatology 38: 178-186, 2003.

24. Fraenzer JT, Pan H, Minimo L Jr, Smith GM, Knauer D and Hung G: Overexpression of the NF2 gene inhibits schwannoma cell proliferation through promoting PDGFR degradation. Int J Oncol 23: 1493-1500, 2003.

25. Pan Y, Wang L and Dai JL: Suppression of breast cancer cell growth by $\mathrm{Na}^{+} / \mathrm{H}^{+}$exchanger regulatory factor 1 (NHERF1). Breast Cancer Res 8: R63, 2006.

26. Cardone RA, Bellizzi A, Busco G, Weinman EJ, Dell'Aquila ME, Casavola V, Azzariti A, Mangia A, Paradiso A and Reshkin SJ: The NHERF1 PDZ2 domain regulates PKA-RhoA-p38-mediated NHE1 activation and invasion in breast tumor cells. Mol Biol Cell 18: 1768-1780, 2007
27. Song J, Bai J, Yang W, Gabrielson EW, Chan DW and Zhang Z: Expression and clinicopathological significance of oestrogenresponsive ezrin-radixinmoesin-binding phosphoprotein 50 in breast cancer. Histopathology 51: 40-53, 2007.

28. Bellizzi A, Malfettone A, Cardone RA and Mangia A: NHERF1/ EBP50 in breast cancer: clinical perspectives. Breast Care (Basel) 5: 86-90, 2010.

29. Bellizzi A, Mangia A, Malfettone A, Cardone RA, Simone G, Reshkin SJ and Paradiso A: $\mathrm{Na}^{+} / \mathrm{H}^{+}$exchanger regulatory factor 1 expression levels in blood and tissue predict breast tumour clinical behaviour. Histopathology 58: 1086-1095, 2011.

30. Mangia A, Chiriatti A, Bellizzi A, Malfettone A, Stea B, Zito FA, et al: Biological role of NHERF1 protein expression in breast cancer. Histopathology 55: 600-608, 2009.

31. Georgescu M, Morales FC, Molina JR and Hayashi Y: Roles of NHERF1/EBP50 in cancer. Curr Mol Med 8: 459-468, 2008.

32. Gebhardt F, Bürger H and Brandt B: Modulation of EGFR gene transcription by secondary structures, a polymorphic repetitive sequence and mutations - a link between genetics and epigenetics. Histol Histopathol 15: 929-936, 2000.

33. Weinman EJ, Steplock D, Wade JB and Shenolikar S: Ezrin binding domain-deficient NHERF attenuates cAMP-mediated inhibition of $\mathrm{Na}(+) / \mathrm{H}(+)$ exchange in $\mathrm{OK}$ cells. Am J Physiol Renal Physiol 281: F374-F380, 2001.

34. Weinman EJ, Wang Y, Wang F, Greer C, Steplock D and Shenolikar S: A C-terminal PDZ motif in NHE3 binds NHERF-1 and enhances CAMP inhibition of sodium-hydrogen exchange. Biochemistry 42: 12662-12668, 2003.

35. Busco G, Cardone RA, Greco MR, Bellizzi A, Colella M, Antelmi E, et al: NHE1 promotes invadopodial ECM proteolysis through acidification of the peri-invadopodial space. FASEB J 24: 3903-3915, 2010.

36. Chambers AF, Groom AC and MacDonald IC: Dissemination and growth of cancer cells in metastatic sites. Nat Rev Cancer 2: 563-572, 2002.

37. Mader CC, Oser M, Magalhaes MA, Bravo-Cordero JJ, Condeelis J, Koleske AJ, et al: An EGFR-Src-Arg-cortactin pathway mediates functional maturation of invadopodia and breast cancer cell invasion. Cancer Res 71: 1730-1741, 2011.

38. Sebastian S, Settleman J, Reshkin SJ, Azzariti A, Bellizzi A and Paradiso A: The complexity of targeting EGFR signalling in cancer: from expression to turnover. Biochim Biophys Acta 1766: 120-139, 2006.

39. Grandal MV and Madshus IH: Epidermal growth factor receptor and cancer: control of oncogenic signalling by endocytosis. J Cell Mol Med 12: 1527-1534, 2008.

40. Lo H-W and Hung M-C: Nuclear EGFR signalling network in cancers: linking EGFR pathway to cell cycle progression, nitric oxide pathway and patient survival. Br J Cancer 94: 184-188, 2006.

41. Greco MR, Antelmi E, Busco G, Guerra L, Rubino R, Casavola V, et al: Protease activity at invadopodial focal digestive areas is dependent on NHE1-driven acidic pHe. Oncol Rep 31: 940-946, 2014. 